\title{
Modelo experimental biológico y molecular de fuerza tensional para el estudio fisiopatológico de la hiperplasia intimal venosa
}

\section{Experimental biological and molecular model of tensile strength for the pathophysiological study of intimal venous hyperplasia}

\author{
Hugo Laparra-Escareño ${ }^{1}$, Alette Ortega-Gómez ${ }^{2}$, José L. Ventura-Gallegos ${ }^{3}$, Alejandro Zentella-Dehesa ${ }^{4}$, \\ Abel Santamaría-del Angel ${ }^{5}$ y Carlos A. Hinojosa ${ }^{1 *}$
}

${ }^{1}$ Departamento de Cirugía Vascular, Instituto Nacional de Ciencias Médicas y Nutrición Salvador Zubirán; '2Laboratorio de Medicina Traslacional, Instituto Nacional de Cancerologí; ${ }^{3}$ Instituto de Investigaciones Biomédicas, Universidad Nacional Autónoma de México; ${ }^{4}$ Unidad de Bioquímica, Instituto Nacional de Ciencias Médicas y Nutrición Salvador Zubirán; ${ }^{5}$ Laboratorio de Aminoácidos, Instituto Nacional de Neurología y Neurocirugía. Ciudad de México, México

\section{Resumen}

Objetivo: La hiperplasia intimal del endotelio venoso es una de las principales causas de trombosis en reconstrucciones vasculares. Este proceso patológico se define como la respuesta celular y molecular caracterizada por una proliferación anormal de células musculares lisas. Se ha descrito una asociación importante de la hiperplasia intimal con señales tróficas, algunas asociadas a factores de crecimiento como el factor de crecimiento derivado de plaquetas. Dichos mecanismos iniciadores aún no han sido bien caracterizados a nivel molecular. La hiperplasia intimal generalmente incluye la presencia de células de músculo liso alfa-actina positivas, matriz extracelular, componentes celulares inflamatorios como macrófagos, leucocitos y una amplia variedad de mediadores y citocinas. El objetivo de este estudio fue desarrollar un modelo experimental para el estudio de la fisiopatología de la hiperplasia intimal venosa en los primeros 7 días. Métodos: Nuestro estudio incluyó 12 conejos. A 6 de estos se les realizó una lesión inducida mediante angioplastia con balón en la vena femoral (fuerza tensional por barotrauma), los restantes fueron controles. Resultados: Utilizando un modelo de lesión tensional venosa por medio de barotrauma pudimos reproducir los eventos detonantes de las primeras fases en el desarrollo de la hiperplasia intimal venosa. Estos cambios se reflejaron a nivel sistémico e histopatológico. Conclusiones: La experiencia del manejo del conejo como modelo experimental cumplió las expectativas técnicas para la evaluación de la fisiopatología de la hiperplasia intimal venosa y esto aunado a la ausencia de complicaciones ( $0 \%$ de mortalidad, $0 \%$ de morbilidad).

Palabras clave: Hiperplasia intimal. PDGF. Modelo de lesión tensional. Vascular. Endotelio venoso.

\section{Abstract}

Objective: Intimal venous endothelial hyperplasia is one of the main causes of thrombosis in vascular reconstructions. This pathological process is defined as the cellular and molecular response characterized by an abnormal proliferation of smooth muscle cells. An important association of intimal hyperplasia with trophic signals has been described, some associated with growth factors such as growth factor derived from platelet-derived growth factor platelets. Such initiating mechanisms have 
Rev Mex Angiol. 2020;48(1)

not yet been well characterized. Intimal hyperplasia generally includes the presence of alpha-actin positive smooth muscle cells, extracellular matrix, inflammatory cellular components such as macrophages, leukocytes and a wide variety of mediators and cytokines. The objective of this study was to develop an experimental model for the study of the pathophysiology of venous intimal hyperplasia in the first 7 days. Methods: Our study included 12 rabbits. 6 of these patients underwent an injury by balloon angioplasty in the femoral vein (tension force by barotrauma), the remaining were controls. Results: Using a model of endothelial lesion through barotrauma, we were able to reproduce the detonating events of the early stages in the development of venous intimal hyperplasia. These changes were reflected at the systemic and histopathological level. Conclusions: The experience of rabbit management as an experimental model met the technical expectations for the evaluation of the pathophysiology of venous intimal hyperplasia and this coupled with the absence of complications $(0 \%$ mortality, $0 \%$ morbidity).

Key words: Intimal hyperplasia. Platelet-derived growth factor. Tension force. Barotrauma. Venous endothelium.

\section{Introducción}

La hiperplasia intimal es la respuesta celular y molecular caracterizada por una proliferación continua y anormal, predominantemente de células musculares lisas, tejido conectivo y matriz extracelular, que conduce a hiperplasia de la capa íntima del vaso y estenosis luminal ${ }^{1,2}$. Varios factores de crecimiento, que estimulan la proliferación celular, son las principales moléculas reguladoras del crecimiento para las células in vitro y probablemente también in vivo ${ }^{3}$. Estos factores de crecimiento se han identificado como precursores en la generación de hiperplasia intimal. Es de particular interés, que el factor de crecimiento derivado de plaquetas (PDGF) ha sido identificado como un marcador de hiperplasia intimal del endotelio vascular. Es importante reconocer la expresión de mediadores durante la patogénesis de la hiperplasia intimal, los cuales incluso pueden ser potenciales blancos terapéuticos $^{5}$. En las fases iniciales del desarrollo de hiperplasia intimal se ha demostrado que los factores de crecimiento constituyen importantes reguladores de migración y proliferación celular ${ }^{2}$. Los factores de crecimiento más importantes de proliferación y migración celular son péptidos que tienen la capacidad de unirse a receptores de tirosina cinasa 6 . Los dos factores de crecimiento más estudiados son PDGF y el factor de crecimiento de fibroblastos (FGF). Existe evidencia de liberación de PDGF durante los primeros minutos posteriores a la manipulación de células endoteliales. El FGF tiene una liberación en etapas más tardías ${ }^{7-9}$.

EI PDGF fue originalmente descrito en sangre, pero también es producido por células musculares lisas y endoteliales. Se trata de una glicoproteína compuesta por dos cadenas de polipéptidos con peso de 27 a 31 kd. El PDGF está formado por moléculas diméricas que pueden existir como homodímeros o heterodímeros de dos cadenas peptídicas diferentes pero relacionadas, llamadas PDGF-A y PDGF-B (es decir, PDGF-AA, PDGF-BB y PDGF-AB). Las células endoteliales secretan de forma predominante, el homodímero PDGF-BB. El PDGF es un importante mitógeno y desempeña un papel importante en la migración de células musculares lisas desde la capa media a la capa íntima ${ }^{10-12}$.

La producción de este factor de crecimiento se ha evaluado experimentalmente. Los injertos venosos implantados en la circulación arterial aumentan de forma significativa la producción de PDGF. Cuando estos injertos son reimplantados en la circulación venosa, la producción disminuye ${ }^{13}$. La administración de antagonistas contra el PDGF en ratas sometidas a angioplastia arterial provocó una reducción del $40 \%$ de hiperplasia intimal ${ }^{14}$. EI PDGF se libera durante los primeros minutos después de la manipulación del endotelio vascular $^{15}$. Es un importante factor quimioatrayente que estimula la migración de las células musculares lisas hacia la capa íntima durante los primeros 7 días después del daño endotelial. Secundario a ello, las células musculares lisas cambian su fenotipo contráctil a uno proliferativo ${ }^{5}$. A partir de entonces, el posterior engrosamiento intimal depende casi exclusivamente de la deposición de matriz extracelular, sobre todo estimulada por el FGF ${ }^{16}$. Debido al importante papel del PDGF en la generación de hiperplasia intimal, la terapia antiplaquetaria se ha propuesto como un modulador en la respuesta endotelial, teniendo como objetivo terapéutico el bloqueo de la activación de las plaquetas $^{17-19}$.

El objetivo de nuestro estudio fue evaluar la hiperplasia de la íntima y su asociación con el PDGF en un modelo experimental (conejo de raza Nueva Zelanda) de lesión tensional (barotrauma) en la vena femoral por medio de una lesión inducida por balón. De esta forma se puede desarrollar un modelo experimental para el estudio de nuevos objetivos terapéuticos en hiperplasia intimal venosa. 


\section{Material y métodos}

\section{Modelo experimental}

Nuestro estudio incluyó 12 conejos de raza Nueva Zelanda, con un peso aproximado de $2.0 \mathrm{~kg}$ cada uno. Se rasuraron las partes laterales dorsales de las orejas y las regiones inguinales. El estudio fue realizado según la Normativa Oficial Mexicana 062-ZOO para el uso y cuidado de animales de laboratorio. Todos los animales fueron operados bajo anestesia general conforme al Comité institucional de cuidado y uso de animales de la Facultad de Medicina de Harvard. Los fármacos empleados fueron: Xilazina $(6 \mathrm{mg} / \mathrm{kg})$, un agonista alfa-adrenérgico A2; ketamina $(50 \mathrm{mg} / \mathrm{kg})$, un inhibidor de $\mathrm{N}$-metil-D-aspartato, pentobarbital (Sagatal) $(40 \mathrm{mg} / \mathrm{kg})$, un anestésico, y paracetamol (100 mg/kg), un antipirético y analgésico.

\section{Procedimiento experimental}

Se realizó un estudio experimental, prospectivo, aleatorizado y controlado, basado en una lesión tensional por barotrauma en la vena femoral. Todos los experimentos se llevaron a cabo en el Departamento de Cirugía de la Universidad Nacional Autónoma de México.

Incluimos un total de 12 conejos machos de la especie Oryctolagus, Nueva Zelanda. Para el desarrollo del modelo incluimos 3 fases:

- Fase I: Se tomó una muestra sanguínea (vena periférica en la oreja derecha). Se realizó una incisión inguinal izquierda (debajo del ligamento inguinal) hasta que se localizó el paquete neurovascular femoral. Se efectuó una punción directa de la vena femoral con un catéter del n. $24 \mathrm{G}$, y se avanzó una guía hidrofílica de 0.14 " hacia la vena cava inferior y la aurícula derecha bajo control fluoroscópico. Utilizando un sistema de rápido intercambio, se avanzó un balón de angioplastia de $20 \times 4 \mathrm{~mm}$. Bajo control fluoroscópico, el balón se colocó en la vena femoral ipsilateral. Se realizó una insuflación a 14 atm de presión (presión de ruptura), para realizar una lesión en la vena femoral secundaria a sobredistensión vascular durante 3 minutos. Mediante este método, se realizó una lesión aguda por barotrauma en el endotelio venoso. La vena femoral en el modelo experimental propuesto mide aproximadamente $1.5 \mathrm{~mm}$, por lo que cuando se usa un balón de $4 \mathrm{~mm}$ se logra distender la vena tres veces su tamaño normal. Finalmente, al retirar el balón se realizó un control angiográfico para verificar la permeabilidad del sistema venoso profundo.
- Fase II: 12 horas después del barotrauma venoso, se obtuvo la segunda muestra de sangre periférica.

- Fase III: 7 días después del procedimiento se tomó la tercera muestra de sangre periférica. Finalmente, los animales fueron sacrificados para estudio histológico y cuantificación de PDGF por ensayo de inmunoabsorción ligado a enzimas (ELISA) (0 horas, 12 horas y 7 días). Para fines de control, se obtuvieron venas femorales contralaterales.

\section{Análisis histopatológico}

Medimos el desarrollo de hiperplasia intimal venosa mediante análisis histopatológico ( 7 días posteriores al barotrauma en el endotelio venoso). El modelo experimental de lesión por balón se comparó con el grupo control. Para este propósito, las muestras de tejido obtenidas se procesaron, se incluyeron en parafina y se seccionaron de acuerdo con métodos estándar usando Tissue-Tek TEC y Tissue-VIP (Sakura Finetek USA, Inc. Torrance, CA, EUA) con un agente de inclusión y un procesador de tejido, respectivamente.

Posteriormente se realizaron tinciones en muestras de $5 \mu \mathrm{m}$ de espesor utilizando hematoxilina y eosina (Leica ST520, Wetzlar, Alemania). Se evaluó y registró la presencia de hiperplasia intimal. Para ello, el área total de las venas se evaluó microscópicamente (Nikoneclipse 80i, Melville, NY, EE.UU.) utilizando la regla estándar en la platina del microscopio. Se realizaron microfotografías de manera sistemática a lo largo de toda la sección de tejido.

\section{Ensayo de densitometría para la evaluación de hiperplasia intimal venosa}

El análisis densitométrico de la hiperplasia intimal en las venas de conejo se evaluó para el grupo experimental (barotrauma) y control usando el software Image J.

\section{Detección de PDGF-BB por ensayo de inmunoabsorción ligado a enzimas}

Las muestras de sangre periférica se obtuvieron a las 0 horas, 12 horas y 7 días. La sangre se mantuvo durante 30 minutos a temperatura ambiente, seguido de 2 horas en hielo para inducir coagulación. Se centrifugaron las muestras a 3,000 revoluciones por minuto, durante 10 minutos para separar el suero, que luego se almacenó a $-80^{\circ} \mathrm{C}$. Para la identificación de proteínas específicas de PDGF-BB a 0 horas, 12 horas y 7 días, utilizamos el kit ELISA RayGioR Rabbit PDFG-BB. 


\section{A}

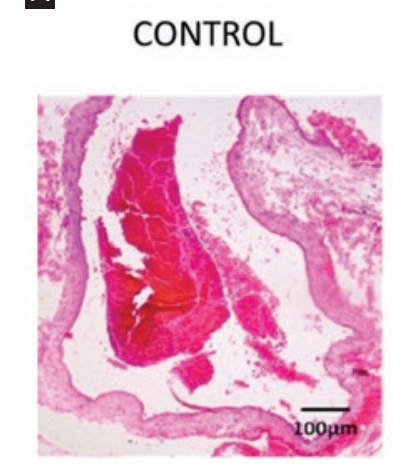

\section{LESIÓN INDUCIDA POR BALÓN}

B

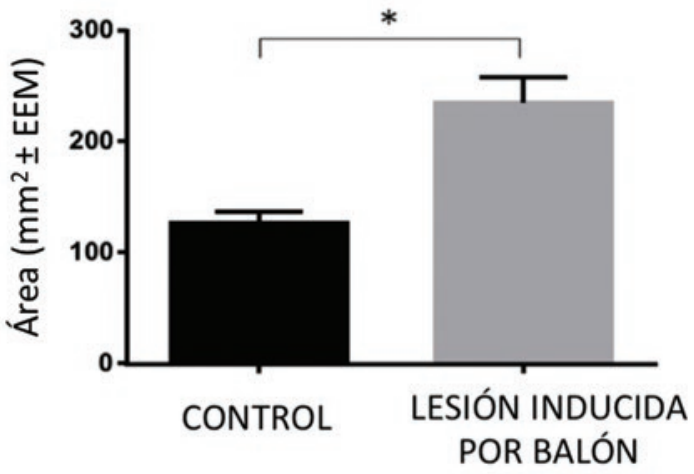

Figura 1. Histoquímica transversal. A: vena control (sin manipulación) y vena con lesión inducida por balón, al séptimo día en cada caso. Escala (barra) $=100 \mu \mathrm{m}$. B: grupo control vs. grupo con lesión inducida por balón, al séptimo día (área $\mathrm{mm}^{2} \pm \mathrm{SEM}$ ). Para el análisis histológico se utilizó imagen de microscopia óptica AXIOVERT. El análisis de densitometría se realizó con el software Image J versión 1.x. Se utilizó una prueba ANOVA de una vía seguida de una prueba post-hoc de Tukey para comparación de las medias. Nivel de significancia ${ }^{*} p \leq 0.05$. SEM: standard error of the mean.

\section{Resultados}

El modelo experimental demostró generar hiperplasia intimal en el endotelio venoso. Este modelo piloto fue factible y reproducible. El conejo de raza Nueva Zelanda como modelo experimental para la hiperplasia intimal cumplió las expectativas técnicas sin complicaciones ( $0 \%$ de mortalidad, $0 \%$ de morbilidad). La hiperplasia intimal producida por el modelo se demostró con el análisis histopatológico.

La medición de la capa íntima en el grupo de venas de control fue $126.12 \mathrm{~mm}^{2} \pm 10.8$, y en el grupo experimental, $232 \mathrm{~mm}^{2} \pm 22.7$. La diferencia entre el grupo control y el grupo experimental fue de $106 \mathrm{~mm}^{2} \pm 11,9$. Al comparar el grupo experimental frente al grupo control, se observó una diferencia estadísticamente significativa (Fig. 1).

En la medición de las concentraciones séricas de PDGF-BB en el grupo experimental, los niveles a las 0 horas fueron de $0.22 \mathrm{pg} / \mathrm{ml} \pm 0.04$. Se observó un pico a las 12 horas de $0,42 \mathrm{pg} / \mathrm{ml} \pm 0,20$, que disminuyó hacia el séptimo día a 0,17 pg/ml $\pm 0,09$ (Fig. 2).

\section{Discusión}

Este trabajo de investigación evalúa los primeros eventos de hiperplasia intimal, centrados en las señales fisiopatológicas secundarias a una lesión vascular por barotrauma sobre un endotelio venoso. Para este fin, hemos desarrollado un nuevo modelo experimental para la evaluación de los cambios histopatológicos de la hiperplasia de la íntima asociada al PDGF como un efector principal. Se evaluó la variante PDGF-BB, ya que es la más específica en el endotelio vascular ${ }^{9,20}$.

Se ha demostrado que uno de los eventos que regulan las fases iniciales de la proliferación de células musculares lisas en la capa media, y su migración a la capa íntima es detonada por el PDGF, el cual se libera de plaquetas y células endoteliales ${ }^{21-23}$. 


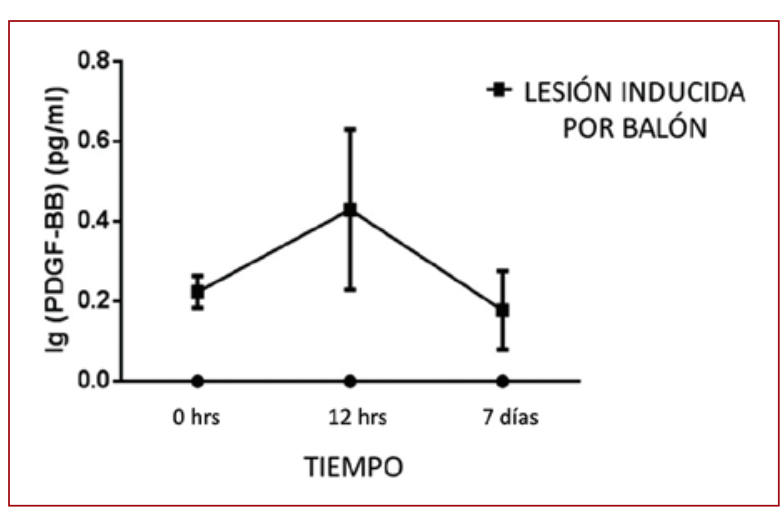

Figura 2. Niveles séricos PDGF-BB dependientes del tiempo después de una lesión inducida por balón. En el suero del grupo experimental, se analizó PDGF-BB (pg/ml) al tiempo 0.12 horas y 7 días. El análisis se realizó en un espectrofotómetro de microplacas Sky Multiscan. Grupo experimental $n=6$ por grupo.

Durante los últimos años se han desarrollado modelos animales para el estudio de la biología vascular. La hiperplasia intimal venosa se ha identificado como una de las principales causas de falla para reconstrucciones vasculares y fístulas arteriovenosas, por lo cual con estos modelos experimentales no solo se ha estudiado la fisiopatología vascular, sino que se han propuesto estrategias terapéuticas para disminuir estas complicaciones en cirugía vascular ${ }^{24}$. Los modelos porcinos, unos de los más utilizados, tienen la ventaja de tener vasos de mayor calibre, accesibles y apegados a la anatomía de un ser humano, sin embargo, su manejo y costo hace que sea difícil su uso cotidiano $^{24,25}$. Los modelos murinos han surgido como un excelente modelo para el estudio de la biología vascular, con el mayor potencial para obtener más información sobre los mecanismos moleculares subyacentes debido a la disponibilidad de tener ratones transgénicos. La principal limitación de este tipo de modelos es el tamaño de los vasos, debido a que se requiere una curva de aprendizaje en técnicas de microcirugía avanzada ${ }^{26,27}$. En rata se desarrolló un modelo de fístula aortocava para estudiar la patogénesis de la hipertrofia cardíaca y el efecto cardiovascular de la insuficiencia cardíaca congestiva. Se ha utilizado, también, para estudiar la fisiopatología de fístulas arteriovenosas ${ }^{25,28}$. Además de compartir limitaciones similares con los ratones, una desventaja de este modelo es que el desarrollo de lesiones estenóticas hemodinámicamente significativas en fístulas arteriovenosas es mucho más lento en ratas que en modelos murinos. Las ratas (sobre todo Wistar) también han demostrado ser un adecuado modelo para estudiar la fisiopatología en la falla de reconstrucciones vasculares ${ }^{29,30}$. Finalmente hay modelos experimentales que, aunque menos frecuentemente usados, también han demostrado su efectividad. Tal es el caso de perros, primates, ovejas y cabras ${ }^{31,32}$. Nosotros elegimos el modelo de conejo para estudiar las alteraciones histopatológicas y sistémicas de la influencia del PDGF en el endotelio venoso posterior a una lesión vascular por barotrauma. Se han descrito algunos experimentos en arterias de conejos, pero esta es la primera descripción de experimentos en venas, específicamente enfocados a hiperplasia intimal. Este modelo experimental demostró generar hiperplasia intimal en el endotelio venoso además de ser reproducible.

Por otro lado, la validez del estudio de alteraciones vasculares en animales ha sido un tema de debate poniendo sobre la mesa las similitudes con pacientes humanos, como por ejemplo estados de insuficiencia renal crónica terminal. Este escepticismo se relaciona principalmente con el uso de modelos animales relativamente sanos que podrían mostrar una respuesta diferente a alteraciones vasculares ${ }^{24}$. Debido a este concepto hay grupos de investigadores que han creado modelos biológicos urémicos con la finalidad de poder replicar las fases más avanzadas de la enfermedad renal crónica terminal y su efecto en fístulas arteriovenosas y reconstrucciones vasculares ${ }^{33}$.

Finalmente, se debe tener en cuenta que la generación de hiperplasia intimal tiene fases y mecanismos fisiopatológicos diferentes, cuyo estudio se debe realizar por separado y el modelo experimental debe ser reproducible en estos escenarios en específico ${ }^{1,2}$. Los cambios hemodinámicos presentes en una anastomosis arteriovenosa son muy complejos y existen diferencias significativas dependiendo del momento y mecanismo de acción de estos eventos fisiológicos y fisiopatológicos ${ }^{6}$.

El paso de sangre a través de un sistema vascular genera dos fuerzas hemodinámicas principalmente: fuerzas de cizallamiento (shear stress), que están directamente relacionadas con la velocidad de flujo, la viscosidad de la sangre y el diámetro del vaso sanguíneo, y las fuerzas de tensión radial, que dependen de la presión de la sangre sobre las dimensiones (diámetro y grosor) del vaso sanguíneo ${ }^{34,35}$. Para estudiar los cambios hemodinámicos en una anastomosis arteriovenosa es muy importante separar ambos mecanismos hemodinámicos debido a que 
son muy diferentes en cuanto a cronología, vías de señalización y fisiopatología. El presente trabajo evalúa las alteraciones más tempranas, enfocadas en el mecanismo fisiopatológico generado por fuerza tensional radial5,16,36.

En estudios experimentales se ha demostrado que las fuerzas tensionales sobre el endotelio venoso en una anastomosis arteriovenosa se elevan más de 10 veces, comparado con el nivel basal. Estos cambios moleculares se pueden detectar de una forma tan temprana como 8 minutos después de la lesión vascu$\operatorname{lar}^{15,37}$. Posterior a una angioplastia con balón en el endotelio venoso se crea una obvia lesión vascular. Esta lesión genera denudación de las células endoteliales. Inmediatamente después de la lesión se adhieren y se granulan plaquetas dentro del vaso afectado. Las células musculares lisas inician su proliferación en la capa media aproximadamente en las primeras 12 horas después de la lesión. Durante los 4 días siguientes, las células musculares lisas migran hacia la capa íntima, donde algunas de ellas continúan proliferando generando estenosis del lumen del vaso ${ }^{36,38,39}$. Posteriormente existe deposición de matriz extracelular alrededor de la capa íntima, lo cual agrava la estenosis. Se ha demostrado que uno de los eventos que regula las fases iniciales de proliferación de células musculares lisas en la capa media y su migración a la capa íntima es detonado por el factor de crecimiento derivado de plaquetas, el cual es liberado de plaquetas y células endoteliales ${ }^{40,41}$. Después de una lesión vascular secundaria a fuerzas de tensión radial, la matriz extracelular se encuentra desorganizada, las células pierden contacto entre ellas y entran en apoptosis. Para compensar aquellas células que sobreviven a la lesión inicial, los factores de crecimiento facilitan el engrosamiento de la capa íntima. Las fuerzas de tensión radial en las etapas más tempranas activan receptores de tirosina cinasa, en donde el PDGF juega un papel fundamental $\left.\right|^{9,20,21,42,43 .,}$ Estos conceptos se demostraron en nuestro modelo experimental.

\section{Conclusiones}

En este trabajo, proponemos un modelo experimental que simula los eventos desencadenantes de la hiperplasia de la íntima, centrado en el PDGF como el principal efector. El modelo experimental fue capaz de generar eventos que detonaron la expresión de PDGF evaluados por ELISA, e hiperplasia de la íntima analizado por histopatología. Por lo tanto, constituye un modelo adecuado para caracterizar vías moleculares centradas en el PDGF e hiperplasia intimal en el endotelio venoso. Es importante reconocer la expresión de mediadores durante la patogénesis de la hiperplasia intimal, los cuales incluso pueden ser potenciales blancos terapéuticos. En las fases iniciales del desarrollo de hiperplasia intimal se ha demostrado que los factores de crecimiento constituyen importantes reguladores de la migración y proliferación celular.

\section{Declaración de ética en animales}

El estudio realizado según la Normativa Oficial Mexicana 062-ZOO para el uso y cuidado de animales de laboratorio. Todos los animales fueron operados bajo anestesia general utilizando el Comité institucional de cuidado y uso de animales de la Facultad de Medicina de Harvard.

\section{Responsabilidades éticas}

Protección de personas y animales. Los autores declaran que los procedimientos seguidos se conformaron a las normas éticas del Comité de experimentación humana responsable y de acuerdo con la Asociación Médica Mundial y la Declaración de Helsinki.

Confidencialidad de los datos. Los autores declaran que han seguido los protocolos de su centro de trabajo sobre la publicación de datos de pacientes.

Derecho a la privacidad y consentimiento informado. Los autores declaran que en este artículo no aparecen datos de pacientes.

\section{Bibliografía}

1. Davies MG, Hagen PO. Pathobiology of intimal hyperplasia.," Br. J. Surg., vol. 81, no. 9, pp. 1254-1269, Sep. 1994.

2. Newby AC, Zaltsman AB. Molecular mechanisms in intimal hyperplasia. J Pathol. 2000;190(3):300-9.

3. McNeil PL, Muthukrishnan L, Warder E, D'Amore PA. Growth factors are released by mechanically wounded endothelial cells. J Cell Biol. 1989;109(2):811-22.

4. Kaiser M, Weyand CM, Björnsson J, Goronzy JJ. Platelet-derived growth factor, intimal hyperplasia, and ischemic complications in giant cell arteritis. Arthritis Rheum. 1998;41(4):623-33.

5. Lemson MS, Tordoir JH, Daemen MJ, Kitslaar PJ. Intimal hyperplasia in vascular grafts. Eur J Vasc Endovasc Surg. 2000;19(4):336-50.

6. Mitra AK, Gangahar DM, Agrawal DK. Cellular, molecular and immunological mechanisms in the pathophysiology of vein graft intimal hyperplasia. Immunol Cell Biol. 2006;84(2):115-24.

7. Hart CE, Kraiss LW, Vergel S, Gilbertson D, Kenagy R, Kirkman T, et al. PDGFbeta receptor blockade inhibits intimal hyperplasia in the baboon. Circulation. 1999;99(4):564-9.

8. Leppänen O, Janjic N, Carlsson MA, Pietras K, Levin C, Vargeese LS, et al. Intimal hyperplasia recurs after removal of PDGF-AB and -BB inhibition in the rat carotid artery injury model. Arterioscler Thromb Vasc Biol. 2000;20(11):89-95.

9. Li L, Blumenthal DK, Terry CM, He Y, Carlson ML, Cheung AK. PDGF-induced proliferation in human arterial and venous smooth muscle cells: molecular basis for differential effects of PDGF isoforms. J Cell Biochem. 2011; 289-98. 
10. Hannink M, Donoghu DJ. Structure and function of platelet-derived growth factor (PDGF) a-nd related proteins. Biochimica et Biophysica Acta (BBA) - Reviews on Cancer. 1989;989(1):1-10.

11. Heldin $\mathrm{CH}$. Structural and functional studies on platelet-derived growth factor. EMBO J. 1992;11(12):4251-9.

12. Tischer E, Gospodarowicz D, Mitchellb R, Silva M, Schilling J, Lau K, et al. Vascular endothelial growth factor: a new member of the platelet-derived growth factor gene family. Biochem Biophys Res Commun 1989;165:3:1198-206.

13. Deguchi J, Namba T, Hamada H, Nakaoka T, Abe J, Sato O, et al Targeting endogenous platelet-derived growth factor B-chain by adenovirus-mediated gene transfer potently inhibits in vivo smooth muscle proliferation after arterial injury. Gene Ther. 1999;6(6):,956-65.

14. Ferns GA, Raines EW, Sprugel KH, Motani AS, Reidy MA, Ross R. Inhibition of neointimal smooth muscle accumulation after angioplasty by an antibody to PDGF. Science. 1991;253(5024):1129-32.

15. Hu Y, Böck G, Wick $G, X u Q$. Activation of PDGF receptor alpha in vascular smooth muscle cells by mechanical stress. FASEB J. 1998;12(12):1135-42.

16. Zubilewicz T, Wronski J, Bourriez A, Terlecki P, Guinault AM, MuscateIli-Groux B. Injury in vascular surgery--the intimal hyperplastic response. Med Sci Monit. 2001;7(2):316-24.

17. Ip JH, Fuster V, Israel D, Badimon L, Badimon J, Chesebro JH. The role of platelets, thrombin and hyperplasia in restenosis after coronary angioplasty. J Am Coll Cardiol. 1991;17(6):77-88.

18. Dixon BS, Beck GJ, Vazquez MA, Greenberg A, Delmez JA, Allon M, et al.; DAC Study Group. Effect of dipyridamole plus aspirin on hemodialysis graft patency. N Engl J Med. 2009: 360(21):2191-201.

19. Dember LM, Beck GJ, Allon M, Delmez JA, Dixon BS, Greenberg A, J, et al.; Dialysis Access Consortium Study Group. Effect of clopidogrel on early failure of arteriovenous fistulas for hemodialysis: a randomized controlled trial. JAMA. 2008;299(18): 2164-71.

20. Cospedal R, Abedi H, Zachary I. Platelet-derived growth factor-BB (PDGF-BB) regulation of migration and focal adhesion kinase phosphorylation in rabbit aortic vascular smooth muscle cells: roles of phosphatidylinositol 3-kinase and mitogen-activated protein kinases. Cardiovasc Res. 1999;41(3):708-21.

21. Yang Z, Oemar BS, Carrel T, Kipfer B, Julmy F, Lüscher TF. Different proliferative properties of smooth muscle cells of human arterial and venous bypass vessels: role of PDGF receptors, mitogen-activated protein kinase, and cyclin-dependent kinase inhibitors. Circulation. 1998;97(2):181-7.

22. Wilson E, Mai Q, Sudhir K, Weiss RH, Ives HE. Mechanical strain induces growth of vascular smooth muscle cells via autocrine action of PDGF. J Cell Biol. 1993;123(3):741-7.

23. Raines EW. PDGF and cardiovascular disease Cytokine Growth Factor Rev. 2004;15(4):237-54.

24. Rotmans Jl. Animal models for studying pathophysiology of hemodialysis access. Open Urol Nephrol. 2014;7(1):14-21.

25. Ferrell M, Fuster V, Gold H.K, Chesebro JH. A dilemma for the 1990s. Choosing appropriate experimental animal model for the prevention of restenosis. Circulation. 1992;85(4):1630-1.
26. Castier Y, Lehoux S, Hu Y, Foteinos G, Tedgui A, Xu Q. Characterization of neointima lesions associated with arteriovenous fistulas in a mouse model. Kidney Int. 2006;70 (2):315-20.

27. Yang B, Shergill U, Fu AA, Knudsen B, Misra S. The mouse arteriovenous fistula model. J Vasc Interv Radiol. 2009;20(7):946-50.

28. Winaver J, Hoffman A, Burnett JC, Haramati A. Hormonal determinants of sodium excretion in rats with experimental high-output heart failure. Am J Physiol. 1988;254(5): R776-84.

29. Chan CY, Chen YS, Ma MC, Chen CF. Remodeling of experimental arteriovenous fistula with increased matrix metalloproteinase expression in rats. J Vasc Surg. 2007;45(4):804-11.

30. Langer S, Heiss C, Paulus N, Bektas N, Mommertz G, Rowinska Z, et al. European Vascular Center Aachen-Maastricht. Functional and structural response of arterialized femoral veins in a rodent AV fistula model. Nephrol Dial Transplant. 2009;24(7):2201-06.

31. Kohler TR, Kirkman TR. Dialysis access failure: A sheep model of rapid stenosis. J Vasc Surg. 1999;30(4):744-51.

32. Lemson MS, Daemen M.J, Kitslaar PJ, Tordoir JH. A new animal model to study intimal hyperplasia in arteriovenous fistulas. J Surg Res. 1999;85(1):51-58.

33. Misra S, Fu AA, Puggioni A, Glockner JF, Rajan DK, McKusick MA. Increased expression of hypoxia-inducible factor-1 alpha in venous stenosis of arteriovenous polytetrafluoroethylene grafts in a chronic renal insufficiency porcine model. J Vasc Interv Radiol. 2008;19(2):260-5.

34. Haruguchi H, Teraoka S. Intimal hyperplasia and hemodynamic factors in arterial bypass and arteriovenous grafts: a review. J Artif Organs. 2003;6(4):227-35

35. Osgood MJ, Hocking KM, Voskresensky IV, Li F D, Komalavilas P, Cheung-Flynn J, et al. Surgical vein graft preparation promotes cellular dysfunction, oxidative stress, and intimal hyperplasia in human saphenous vein. J Vasc Surg. 2014;60(1):202-11.

36. Clowes AV. Intimal hyperplasia and graft failure. Chapter 17. Cardiovasc Pathol. 1993;2(3):179-86.

37. Lehoux S, Castier Y, Tedgui A. Molecular mechanisms of the vascular responses to haemodynamic forces. J Intern Med. 2006;259(4):381-92.

38. Weiss MF, Scivittaro V, Anderson JM. Oxidative stress and increased expression of growth factors in lesions of failed hemodialysis access. Am J Kidney Dis. 2001;37(5):970-80.

39. Ward AO, Caputo M, Angelini GD, George SJ, Zakkar M. Activation and inflammation of the venous endothelium in vein graft disease. Atherosclerosis. 2017;265:266-74.

40. Turner NA, Ho S, Warburton P, O'Regan DJ, Porter KE. Smooth muscle cells cultured from human saphenous vein exhibit increased proliferation, invasion, and mitogen-activated protein kinase activation in vitro compared with paired internal mammary artery cells. J Vasc Surg. 2007; 45(5):1022-8.

41. Fuster V, Chesebro JH. Role of platelets and platelet inhibitors in aortocoronary artery vein-graft disease. Circulation. 1986;73(2,):227-32.

42. Fredriksson L, Li H, Eriksson U. The PDGF family: four gene products form five dimeric isoforms. Cytokine Growth Factor Rev. 2004;15(4):197-204.

43. Hirst SJ, Barnes PJ, Twort CH. PDGF isoform-induced proliferation and receptor expression in human cultured airway smooth muscle cells. Am J Physiol. 1996;270(3)1:L415-28. 